\title{
Subsequent systemic therapy for non-small cell lung cancer patients with immune checkpoint inhibitor-related interstitial lung disease
}

\author{
Yusuke Sato $^{1}$, Satoshi Watanabe ${ }^{1}$, Takeshi Ota ${ }^{2}$, Kohei Kushiro ${ }^{1}$, Toshiya Fujisaki ${ }^{1}$, Miho Takahashi ${ }^{1}$, \\ Aya Ohtsubo ${ }^{1}$, Satoshi Shoji ${ }^{1}$, Koichiro Nozaki ${ }^{1}$, Kosuke Ichikawa ${ }^{1}$, Satoshi Hokari ${ }^{1}$, Rie Kondo ${ }^{1}$, \\ Masachika Hayashi ${ }^{1}$, Hiroyuki Ishikawa ${ }^{3}$, Takao Miyabayashi ${ }^{4}$, Tetsuya Abe ${ }^{4}$, Satoru Miura ${ }^{5}$, \\ Hiroshi Tanaka $^{5}$, Masaaki Okajima ${ }^{6}$, Masaki Terada $^{6}$, Takashi Ishida ${ }^{7}$, Akira Iwashima ${ }^{8}$, Kazuhiro Sato ${ }^{9}$, \\ Hirohisa Yoshizawa ${ }^{10}$, Nobumasa Aoki ${ }^{1}$, Yasuyoshi Ohshima ${ }^{1}$, Toshiyuki Koya ${ }^{1}$, Toshiaki Kikuchi ${ }^{1}$ \\ ${ }^{1}$ Department of Respiratory Medicine and Infectious Diseases, Niigata University Graduate School of Medical and Dental Sciences, Niigata, Japan; \\ ${ }^{2}$ Niigata Prefectural Shibata Hospital, Niigata, Japan; ${ }^{3}$ Department of Radiology and Radiation Oncology, Niigata University Graduate School \\ of Medical and Dental Sciences, Niigata, Japan; ${ }^{4}$ Niigata City General Hospital, Niigata, Japan; ${ }^{5}$ Niigata Cancer Center Hospital, Niigata, Japan; \\ ${ }^{6}$ Saiseikai Niigata Hospital, Niigata, Japan; ${ }^{7}$ Niigata Prefectural Central Hospital, Joetsu, Japan; ${ }^{8}$ Nagaoka Chuo General Hospital, Nagaoka, Japan; \\ ${ }^{9}$ Nagaoka Red Cross Hospital, Nagaoka, Japan; ${ }^{10}$ Niigata Medical Center, Niigata, Japan \\ Contributions: (I) Conception and design: Y Sato, S Watanabe, T Ota, M Okajima, S Miura, H Tanaka, T Kikuchi; (II) Administrative support: \\ S Watanabe, H Yoshizawa, T Kikuchi; (III) Provision of study materials or patients: All authors; (IV) Collection and assembly of data: Y Sato, \\ M Okajima, S Miura, S Watanabe; (V) Data analysis and interpretation: T Ota, M Okajima, S Miura, S Watanabe, H Tanaka, T Kikuchi; (VI) \\ Manuscript writing: All authors; (VII) Final approval of manuscript: All authors. \\ Correspondence to: Dr. Satoshi Watanabe. Department of Respiratory Medicine and Infectious Diseases, Niigata University Graduate School of \\ Medical and Dental Sciences, 1-757 Asahimachidori, Chuouku, Niigata, 951-8510, Japan. Email: satoshi7@med.niigata-u.ac.jp.
}

Background: Although immune checkpoint inhibitors (ICIs) are effective for advanced non-small cell lung cancer (NSCLC), ICIs may cause interstitial lung disease (ILD), which results in treatment discontinuation and is sometimes fatal. Despite the high incidence of ICI-related ILD, there are few cancer treatment options for patients. This study aimed to evaluate the safety and efficacy of subsequent systemic cancer therapy in NSCLC patients with ICI-related ILD.

Methods: We retrospectively assessed NSCLC patients who received programmed cell death-1 (PD-1) inhibitors as first- to third-line therapy at participating institutions of the Niigata Lung Cancer Treatment Group from January 2016 to October 2017.

Results: This analysis included 231 patients, 32 (14\%) of whom developed ICI-related ILD. Of these patients, 16 (7\%) received subsequent systemic cancer treatments. The median overall survival (OS) tended to be longer in the systemic cancer therapy group than in the no systemic cancer therapy group [22.2 months (95\% CI: 1-NE) vs. 4.5 months (95\% CI: 1-NE); P=0.067]. ICI-related ILD recurred in half of the patients who received systemic cancer therapy, and the median OS tended to be shorter in patients with recurrent ICI-related ILD [22.0 months (95\% CI: 1-NE) vs. 7.0 months (95\% CI: 1-NE); P=0.3154].

Conclusions: According to the current study, systemic cancer treatment is effective in patients with ICIrelated ILD; however, its safety is uncertain because of the high risk of ICI-related ILD recurrence and poor survival outcome following ILD recurrence.

Keywords: Non-small cell lung cancer (NSCLC); PD-1; PD-L1; interstitial lung disease (ILD); immune-related adverse event (irAE)

Submitted Mar 11, 2021. Accepted for publication Jun 15, 2021.

doi: $10.21037 /$ tlcr-21-198

View this article at: https://dx.doi.org/10.21037/tlcr-21-198 


\section{Introduction}

Immune checkpoint inhibitors (ICIs) have emerged as a promising treatment for advanced-stage lung cancer. In particular, programmed cell death-1 (PD-1) and PD-ligand 1 (PD-L1) antibodies show excellent therapeutic effects in terms of improving overall survival (OS) and progressionfree survival (PFS) in patients with advanced-stage lung cancer (1-5). However, ICIs cause immune-related adverse events (irAEs) because these drugs act on the immune system (6). IrAEs occur in various organs, and skin rash, endocrine toxicities and liver dysfunction are particularly frequent (7). Since irAEs could be severe and even fatal, some patients are required to discontinue ICIs. On the other hand, previous studies have indicated that patients who discontinue ICIs due to irAEs have a worse prognosis than those who continue ICIs (8).

Previous studies have also reported that the antitumor effects of ICIs are augmented in patients with irAEs $(9,10)$. Furthermore, the therapeutic effects of cytotoxic chemotherapies are enhanced after ICI treatment $(11,12)$. Readministration of ICIs and cytotoxic chemotherapies seems to be beneficial for patients who discontinue ICIs due to irAEs. By contrast, both ICIs and chemotherapies in patients with irAEs increase the risk of irAE recurrence. Simonaggio and colleagues reported that $55 \%$ of patients who received rechallenge with ICIs experienced recurrence with the same or different irAEs (13). The second irAE was not worse than the first, and they concluded that readministration of ICIs was acceptable in patients who discontinued ICIs due to irAEs.

ICI-related interstitial lung disease (ILD) often leads to treatment discontinuation and is sometimes fatal $(1,8)$. We previously demonstrated that the prognosis of patients with ICI-related ILD was worse than that of patients with other irAEs, and there is a correlation between prognosis and the radiological findings of ILD (14). Previous studies have also demonstrated that cytotoxic chemotherapies and tyrosine kinase inhibitors (TKIs) are risk factors for ILD recurrence (15-17). However, data on the risk of ICI-related ILD recurrence and the prognosis of patients with ICI-related ILD after subsequent systemic cancer therapy are lacking.

In the present study, we performed a retrospective analysis to determine the safety and efficacy of systemic cancer therapy in non-small cell lung cancer (NSCLC) patients with ICI-related ILD.

We present the following article in accordance with the STROBE reporting checklist (available at https://dx.doi. org/10.21037/tlcr-21-198).

\section{Methods}

\section{Study design and patients}

We retrospectively analyzed consecutive patients who received PD-1 inhibitor treatment as first- to third-line therapy at participating institutions of the Niigata Lung Cancer Treatment Group from January 2016 to October 2017. The study was conducted in accordance with the Declaration of Helsinki (as revised in 2013). The study was approved by institutional review board of Niigata University (the registration number: 2017-0295) and each participating institution. Individual consent for this retrospective analysis was waived.

\section{Study assessment}

All patient data were collected retrospectively, and ICIrelated ILD patients were considered. In this study, we investigated the safety and efficacy of chemotherapy and ICI treatment as posttreatment based on OS, the treatment duration, the tumor response, and radiological features. ICI-related ILD was diagnosed by the attending physician at each institution, and chest CT scans were reviewed by two independent respiratory physicians and one radiologist. Regardless of whether patients had interstitial lung abnormality at baseline, we diagnosed ICI-related ILD if patients had pneumonitis after the initiation of anti-PD-1 therapy. ICI-related ILD was graded according to the Common Terminology Criteria for Adverse Events version 4.0. OS was defined as the time between the start date of anti-PD-1 therapy and death from any cause.

\section{Statistical analysis}

We created Kaplan-Meier OS curves for both groups and tested for significant differences with the log-rank test. To minimize lead-time bias associated with time-dependent factors, we performed landmark analysis including only patients who were alive or whose ICI-related ILD was under control at 6 weeks after the onset of initial ICIrelated ILD $(\mathrm{n}=30)$. Continuous variables are presented as the median (range) and were compared by 2 -sided $t$-tests. Categorical variables were compared by Fisher's exact test or the chi-square test. All the reported $\mathrm{P}$ values are 2 -sided, and $\mathrm{P}<0.05$ was considered significant. Statistical analysis 


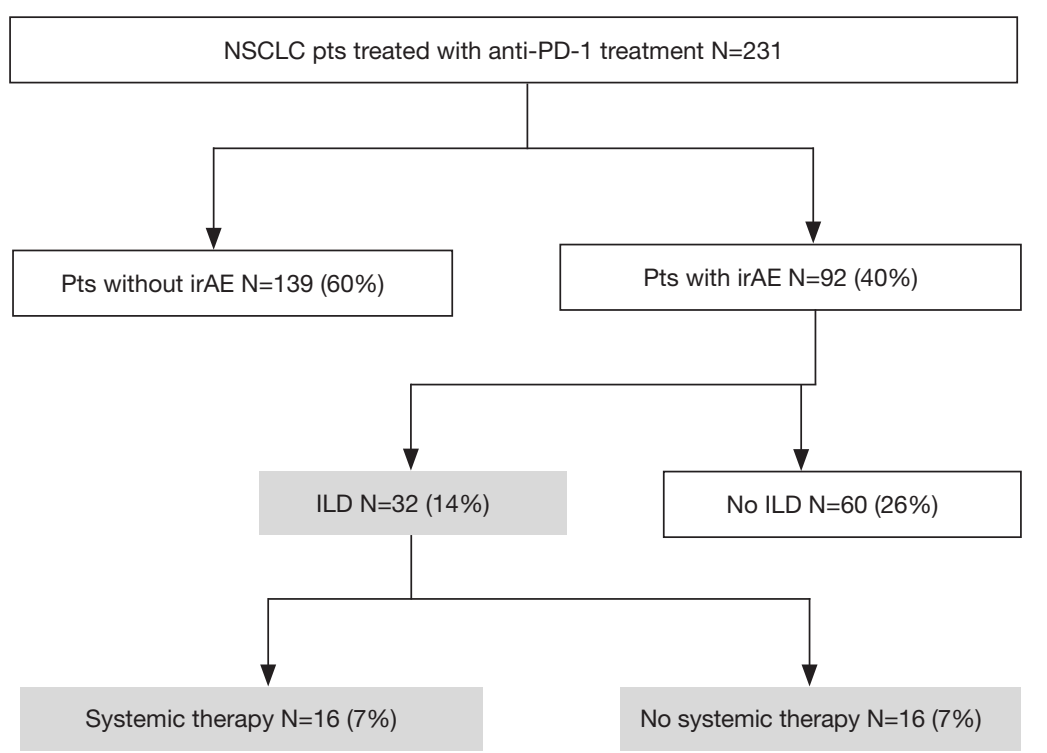

Figure 1 Patient flow diagram. NSCLC, non-small cell lung cancer; PD-1, programmed cell death-1; Pts, patients; irAE, immune-related adverse event; ILD, interstitial lung disease.

was performed using JMP 9.0.2 statistical software (SAS Institute, Cary, NC, USA).

\section{Results}

\section{Characteristics of the study population}

The current study included 231 patients and the observation period for this study was from January 2016 to November 2019, 32 (14\%) of whom developed ICI-related ILD during anti-PD-1 therapy. Of these patients, $16(50 \%)$ received subsequent systemic cancer treatment, and $16(50 \%)$ did not receive systemic cancer treatment after anti-PD-1 therapy (Figure 1). Table 1 shows the baseline characteristics of patients with or without subsequent systemic cancer therapy at the start of anti-PD-1 therapy. No significant differences were observed in terms of age, sex, smoking status, Eastern Cooperative Oncology Group (ECOG) performance status, disease stage, histology, line of anti-PD-1 therapy, PD-L1 expression, type of anti-PD-1 therapy, initial response to anti-PD-1 therapy, baseline interstitial lung abnormality, grade of the initial episode of ICI-related ILD, or radiological features.

\section{OS with subsequent systemic cancer treatment}

Kaplan-Meier OS curves of the groups with and without subsequent systemic cancer treatment are shown in Figure 2.
The median OS tended to be longer in the systemic cancer therapy group than in the no systemic cancer therapy group [22.2 months (95\% CI: $1-\mathrm{NE})$ vs. 4.5 months (95\% CI: $1-\mathrm{NE}$ ); $\mathrm{P}=0.067]$. To minimize immortal time bias, we performed 6-week landmark analysis $(n=30$, Figure S1). Median OS in patients receiving subsequent cancer therapy was tended to be longer than that in patients who did not receive subsequent cancer therapy [22.0 months (95\% CI: 7.3-NE) vs. 5.5 months (95\% CI: 2.2-NE); $\mathrm{P}=0.165]$.

\section{Subsequent systemic cancer treatment regimens}

Of the 16 patients who received subsequent systemic cancer therapy, 8 (9 regimens) developed recurrent ICI-related ILD (Table 2). There were no significant differences in patient characteristics between patients with and without the recurrence of ICI-related ILD (Table S1). The grade of recurrent ICI-related ILD was 2 or less in 7 of 9 regimens (78\%). The grade of the first episode of ILD was 2 or less in 11 of 16 patients (69\%), and the recurrent episode of ICIrelated ILD was not more severe than the first $(\mathrm{P}=0.629)$. All patients were treated with methylprednisolone (mPSL) pulse therapy and/or prednisolone (PSL). No patient died of ICI-related ILD, but one patient died of the progression of NSCLC. Other 7 patients responded to steroid therapies and recovered from the recurrence of ICI-related ILD. Regimens in the ILD recurrence group were S-1, docetaxel 
Table 1 Baseline characteristics

\begin{tabular}{|c|c|c|c|}
\hline Characteristic & Subsequent systemic therapy & No systemic therapy & $P$ value \\
\hline Median age (range), years & $65[45-74]$ & 67 [59-82] & $0.091^{\mathrm{a}}$ \\
\hline Sex, n (\%) & & & $0.37^{\mathrm{b}}$ \\
\hline Male & $12[75]$ & $14[87]$ & \\
\hline Smoking status, $\mathrm{n}(\%)$ & & & $0.29^{b}$ \\
\hline Current or former & $13[81]$ & 15 [94] & \\
\hline Never & $3[19]$ & $1[6]$ & \\
\hline ECOG-PS, n (\%) & & & $0.19^{b}$ \\
\hline$\geq 2$ & $1[6]$ & $5[31]$ & \\
\hline Unknown & $1[6]$ & 0 & \\
\hline Disease stage, $\mathrm{n}(\%)$ & & & $0.25^{\mathrm{b}}$ \\
\hline IIIB & 0 & $1[6]$ & \\
\hline IV & $8[50]$ & $11[69]$ & \\
\hline Relapse after local therapy & $8[50]$ & $4[24]$ & \\
\hline Histology, n (\%) & & & $0.31^{\mathrm{b}}$ \\
\hline Adenocarcinoma & $4[25]$ & $8[50]$ & \\
\hline 3 & $5[31]$ & $5[31]$ & \\
\hline PD-L1 expression, $n$ (\%) & & & $0.19^{b}$ \\
\hline$<1$ & $1[6]$ & 0 & \\
\hline $1-49 \%$ & 0 & $1[6]$ & \\
\hline$\geq 50 \%$ & $2[13]$ & $6[38]$ & \\
\hline Unknown & $13[81]$ & $9[56]$ & \\
\hline Anti-PD-1 therapy, n (\%) & & & $0.10^{\mathrm{b}}$ \\
\hline Nivolumab & 14 [88] & 10 [63] & \\
\hline Pembrolizumab & $2[13]$ & $6[38]$ & \\
\hline Response to anti-PD- 1 therapy & & & $0.63^{\mathrm{b}}$ \\
\hline
\end{tabular}

Table 1 (continued) 
Table 1 (continued)

\begin{tabular}{|c|c|c|c|}
\hline Characteristic & Subsequent systemic therapy & No systemic therapy & $P$ value \\
\hline $\mathrm{CR}$ & $2[13]$ & $1[6]$ & \\
\hline PR & $4[25]$ & $5[31]$ & \\
\hline SD & $6[38]$ & $3[19]$ & \\
\hline $\mathrm{NE}$ & $1[6]$ & $3[19]$ & \\
\hline Baseline interstitial lung abnormality, $\mathrm{n}(\%)$ & $3[19]$ & 0 & $0.07^{\mathrm{b}}$ \\
\hline Grade of initial ILD & & & $0.69^{b}$ \\
\hline $1-2$ & $11[69]$ & $12[75]$ & \\
\hline COP-like & $9[56]$ & $7[44]$ & \\
\hline GGO & $7[44]$ & $8[50]$ & \\
\hline Not otherwise specified & 0 & $1[6]$ & \\
\hline Systemic steroid use, n (\%) & $13[81]$ & $11[69]$ & $0.43^{b}$ \\
\hline Time to the ILD, days (range) & $42(1-523)$ & $38(5-340)$ & $0.97^{\mathrm{a}}$ \\
\hline
\end{tabular}

Differences between groups were identified using ${ }^{2}$ student's $t$-test or ${ }^{b} \mathrm{Chi}$-Square test. ECOG, Eastern Cooperative Oncology Group; PS, performance status; PD-1, programmed cell death-1; PD-L1, PD-ligand 1; CR, complete response; PR, partial response; SD, stable disease; PD, progressive disease; NE, not evaluable; ILD, interstitial lung disease; COP, cryptogenic organizing pneumonia; GGO, ground glass opacity.

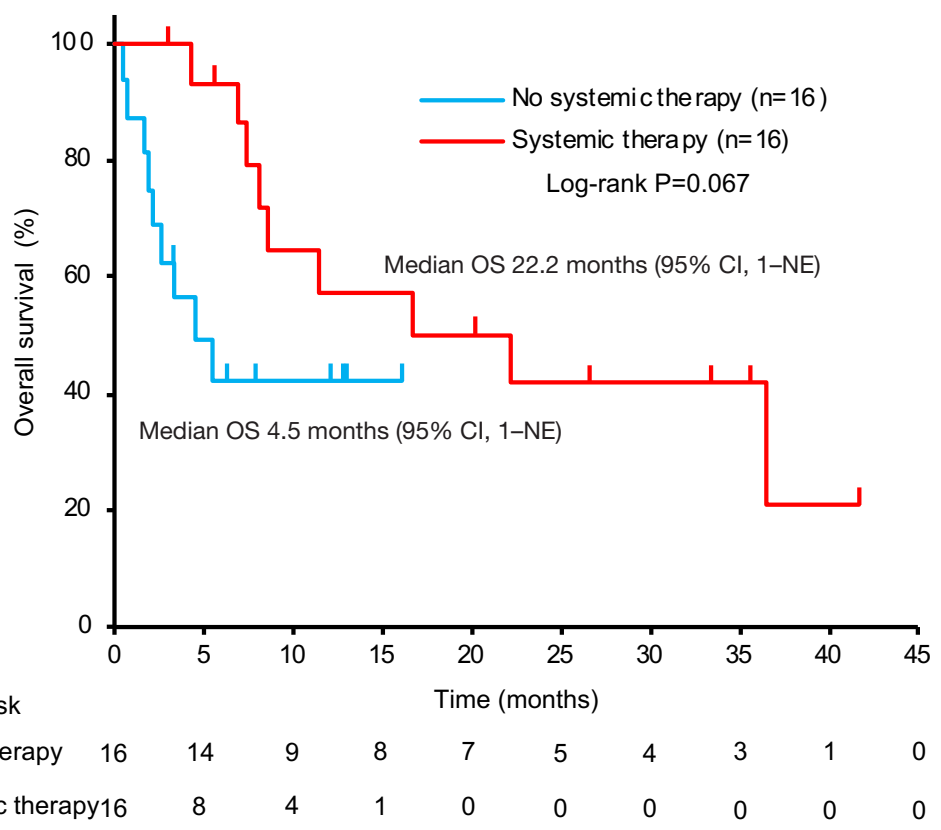

Figure 2 Overall survival curves of patients with or without systemic cancer therapy after the 1st episode of ICI-related ILD. OS, overall survival; CI, confidence interval; NE, not evaluable; ICI, immune checkpoint inhibitor; ILD, interstitial lung disease. 
Table 2 Demographics of patients with recurrent ICI-related ILD $(\mathrm{n}=8)$

\begin{tabular}{lc}
\hline Clinical features & ILD recurrence $(\mathrm{N}=8)$ \\
\hline $\begin{array}{l}\text { Duration of systemic cancer therapy, } \\
\text { days [range] }\end{array}$ & $5[1-183]$ \\
Treatment cycles, median [range] & $1[1-14]$ \\
Grade of ILD, $\mathrm{n}$ [\%] & \\
1 & $1[11]$ \\
2 & $6[67]$ \\
3 & $1[11]$ \\
4 & $1[11]$ \\
5 & 0 \\
Systemic steroid therapy, $\mathrm{n}[\%]$ & \\
$\mathrm{mPSL}$ pulse & $4[44]$ \\
$\mathrm{PSL} \geq 30$ mg/day & $3[33]$ \\
$\mathrm{PSL}<30$ mg/day & $2[22]$ \\
\hline
\end{tabular}

$\mathrm{ICI}$, immune checkpoint inhibitor; ILD, interstitial lung disease; PSL, prednisolone; mPSL, methylprednisolone.

Table 3 Regimens of systemic therapy

\begin{tabular}{lcc}
\hline Regimen & $\begin{array}{c}\text { ILD recurrence } \\
(\mathrm{N}=8)\end{array}$ & $\begin{array}{c}\text { No ILD recurrence } \\
(\mathrm{N}=8)\end{array}$ \\
\hline S-1, n [\%] & $2[22]$ & $3[13]$ \\
DTX+RAM, n [\%] & $2[22]$ & $2[8]$ \\
Nivolumab, n [\%] & $2[22]$ & $2[8]$ \\
DTX, n [\%] & $1[10]$ & $3[13]$ \\
Atezolizumab, n [\%] & $1[10]$ & $2[8]$ \\
CBDCA+VNR, n [\%] & $1[10]$ & 0 \\
CBDCA+S-1, n [\%] & 0 & $3[13]$ \\
VNR, n [\%] & 0 & $2[8]$ \\
nab-PTX, n [\%] & 0 & $2[8]$ \\
Amrubicin, $\mathrm{n}[\%]$ & 0 & $1[4]$ \\
CDDP+PEM, n [\%] & 0 & $1[4]$ \\
Nedaplatin, n [\%] & 0 & $1[4]$ \\
Irinotecan, n [\%] & 0 & $1[4]$ \\
Gemcitabine, n [\%] & 0 & $1[4]$ \\
Total number of regimens & 9 & 24 \\
\hline
\end{tabular}

DTX, docetaxel; RAM, ramucirumab; CBDCA, carboplatin; VNR, vinorelbine; nab-PTX, nab-paclitaxel; CDDP, cisplatin; PEM, pemetrexed; ILD, interstitial lung disease. plus ramucirumab, and nivolumab [in 2 patients each $(22 \%)]$ and docetaxel, atezolizumab, and carboplatin plus vinorelbine [in one patient each (11\%)] (Table 3).

\section{Tumor responses to systemic cancer therapy}

Kaplan-Meier OS curves of the groups with and without ICI-related ILD recurrence are shown in Figure 3. The median OS was longer in the ILD nonrecurrence group than in the ILD recurrence group [22.0 months (95\% CI: $1-\mathrm{NE}$ ) vs. 7.0 months (95\% CI: $1-\mathrm{NE}$ ); $\mathrm{P}=0.3154]$. Table 4 demonstrates tumor responses to systemic cancer therapy in patients with and without ICI-related ILD recurrence. There were no significant differences in overall response rate $(10 \%$ vs. $8 \%, \mathrm{P}=0.88)$ and disease control rate ( $30 \%$ vs. $54 \%, \mathrm{P}=0.47$ ) between two groups.

Figure 4 shows the comparison of radiological features between the first episode of ICI-related ILD and the second and subsequent episodes of ILD. Eight of nine patients with ILD recurrence had the same radiologic features between the first and second episodes of ILD. Only one patient with cryptogenic organizing pneumonia (COP) had a groundglass opacity (GGO) at recurrence.

The ILD nonrecurrence group did better than the ILD recurrence group in terms of the disease control rate $(54 \%$ vs. $22 \%, \mathrm{P}=0.029)$. The overall response rate was similar between groups ( $8 \%$ vs. $11 \%)$.

\section{Duration of treatment}

The Swimmer's plot shows the duration of treatment after the start of subsequent systemic cancer treatment (Figure 5). All patients stopped subsequent therapies when ICI-related ILD relapsed. Two patients in the ILD recurrence group who were rechallenged with ICIs had sustained therapeutic effects at the data cutoff even after ICI discontinuation.

\section{Discussion}

NSCLC patients with ILD including ILD induced by systemic cancer therapy had poor survival outcomes (18). Although the development of tyrosine-kinase inhibitors and ICIs dramatically improved the survival of NSCLC patients, poor outcomes have been reported in patients with ILD related to TKIs and ICIs $(19,20)$. It remains unclear how to treat NSCLC patients with drug-induced ILD. The current study demonstrated that patients who received subsequent 


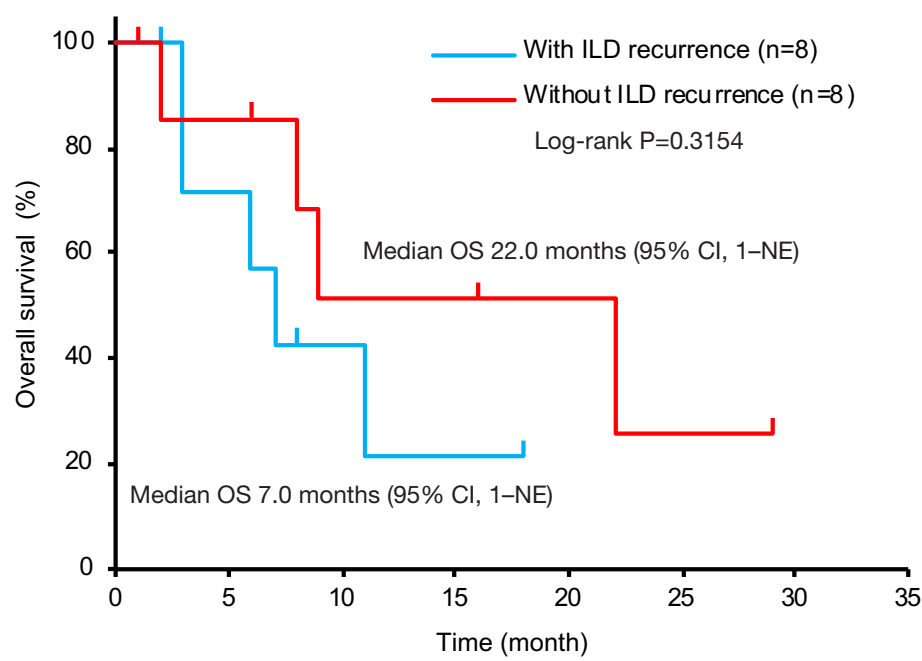

No. at risk

$\begin{array}{lllllllll}\text { Without ILD relapse } & 8 & 6 & 8 & 3 & 2 & 1 & 0 & 0 \\ \text { With ILD relapse } & 8 & 5 & 2 & 2 & 0 & 0 & 0 & 0\end{array}$

Figure 3 Overall survival curves of patients with or without ILD recurrence after systemic cancer therapy. ILD, interstitial lung disease; OS, overall survival; CI, confidence interval; NE, not evaluable.

Table 4 Tumor responses to systemic therapy

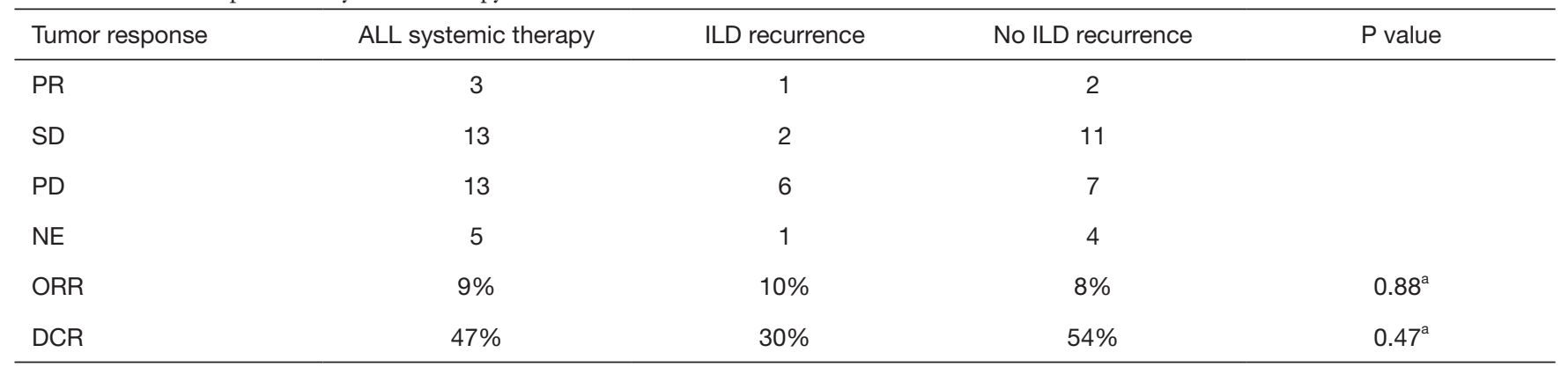

Differences between groups were identified using ${ }^{\mathrm{a} C h i-S q u a r e ~ t e s t . ~ I L D, ~ i n t e r s t i t i a l ~ l u n g ~ d i s e a s e ; ~ P R, ~ p a r t i a l ~ r e s p o n s e ; ~ S D, ~ s t a b l e ~ d i s e a s e ; ~}$ $\mathrm{PD}$, progressive disease; NE, not evaluable; ORR, overall response rate; DCR, disease control rate.

systemic cancer therapy had a better prognosis than those who did not receive systemic cancer therapy after the onset of ICI-related ILD. Recent meta-analyses revealed that patients treated with PD-1 inhibitors had a significantly higher incidence of drug-induced ILD than those treated with chemotherapy. Su et al. showed that PD-1 inhibitors significantly increased grade $1-5$ and grade $3-5$ pneumonitis (risk ratio: 5.17, 95\% CI: 2.82-9.47, $\mathrm{P}<0.001$; risk ratio, 4.14, 95\% CI: $1.82-9.42, \mathrm{P}<0.001)(17)$. Huang et al. also demonstrated that the odds ratios (ORs) of immune-related all-grade and high-grade pneumonitis were significant for nivolumab (all-grade: OR =6.29, 95\% CI: 2.67-16.75; highgrade: $\mathrm{OR}=5.95,95 \%$ CI: $2.35-17.29)$ and pembrolizumab (all-grade: $\mathrm{OR}=5.78,95 \%$ CI: 2.79-13.24; highgrade: $\mathrm{OR}=5.33,95 \%$ CI: 2.49-12.97) compared with chemotherapy (21). Indeed, the current study showed that ICI-related ILD developed in 32 of 231 NSCLC patients (14\%, Figure 1). Despite the high risk of ILD development, there are few cancer treatment options for NSCLC patients with ICI-related ILD. The efficacy and safety of posttreatment for drug-induced ILD that developed following epidermal growth factor receptor (EGFR) 


\begin{tabular}{l|cc}
\multicolumn{1}{l|}{ Radiologic features of ILD recurrence } & 1st ILD \\
& GGO $(\mathrm{N}=4)$ & COP-like $(\mathrm{N}=4)$ \\
\hline 2nd or 3rd ILD & 5 & 1 \\
COP-like & 0 & 3
\end{tabular}

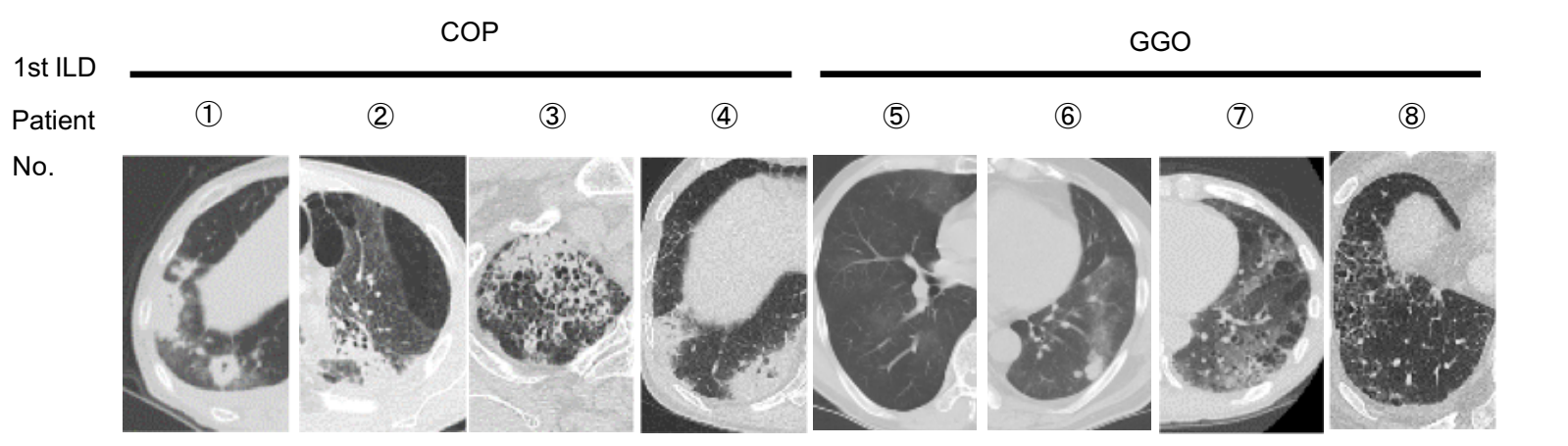

2nd or 3rd ILD

Patient

No.

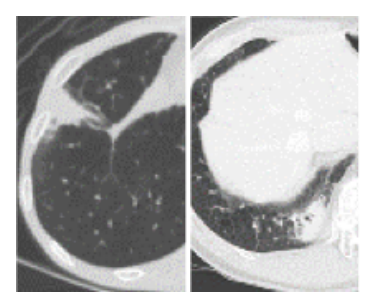

COP

(2)

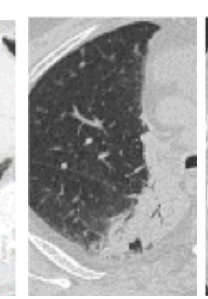

(4)

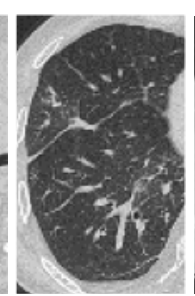

GGO

(5)

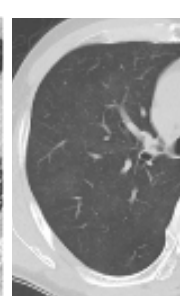

(6)

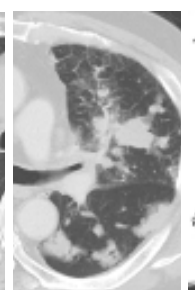

(7)

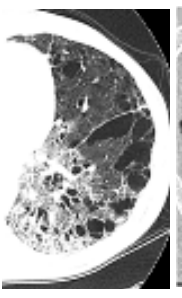

(8)a

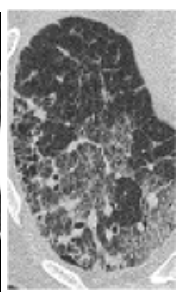

(8) b

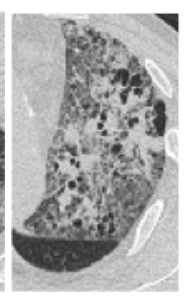

Figure 4 Comparison of radiologic features between the 1st and 2nd episodes of drug-induced ILD. Patient number 8 had 2 recurrences post-ILD treatment. ILD, interstitial lung disease; GGO, ground glass opacity; COP, cryptogenic organizing pneumonia.

TKIs and chemotherapy have been shown in existing reports $(22,23)$. The median OS from the occurrence of chemotherapy-induced ILD tended to be longer in patients who received subsequent chemotherapy than in patients who did not (7.3 vs. 1.9 months, $\mathrm{P}=0.233$ ) (22). Similarly, the median OS from the occurrence of EGFR-TKIinduced ILD was longer in patients who were rechallenged with EGFR-TKIs than in those who were not $(15.5 \mathrm{vs}$. 3.5 months, $\mathrm{P}=0.029$ ) (23). Furthermore, rechallenge with EGFR-TKIs was well tolerated even in patients with EGFR-TKI-induced ILD. In the current study, we showed that OS in patients who received subsequent systemic cancer therapies, including ICIs and cytotoxic chemotherapies, tended to be longer than that in patients who did not receive subsequent therapies (Figure 2).

Systemic cancer therapy in patients with ICI-related ILD is associated with a high risk of ILD recurrence. Simonaggio et al. demonstrated that ICI retreatment after the initial irAE resulted in a second irAE in $55 \%$ of patients (13). They also showed that the severity of the second irAE was not worse than that of the first irAE (13). The current study demonstrated that 8 of 16 patients (50\%) with ICI-related ILD experienced ILD recurrence after sequential systemic cancer therapy (Table 2). There is a possibility that the recurrence of ILD was due to the natural history of ICI-related ILD; however, all but one 


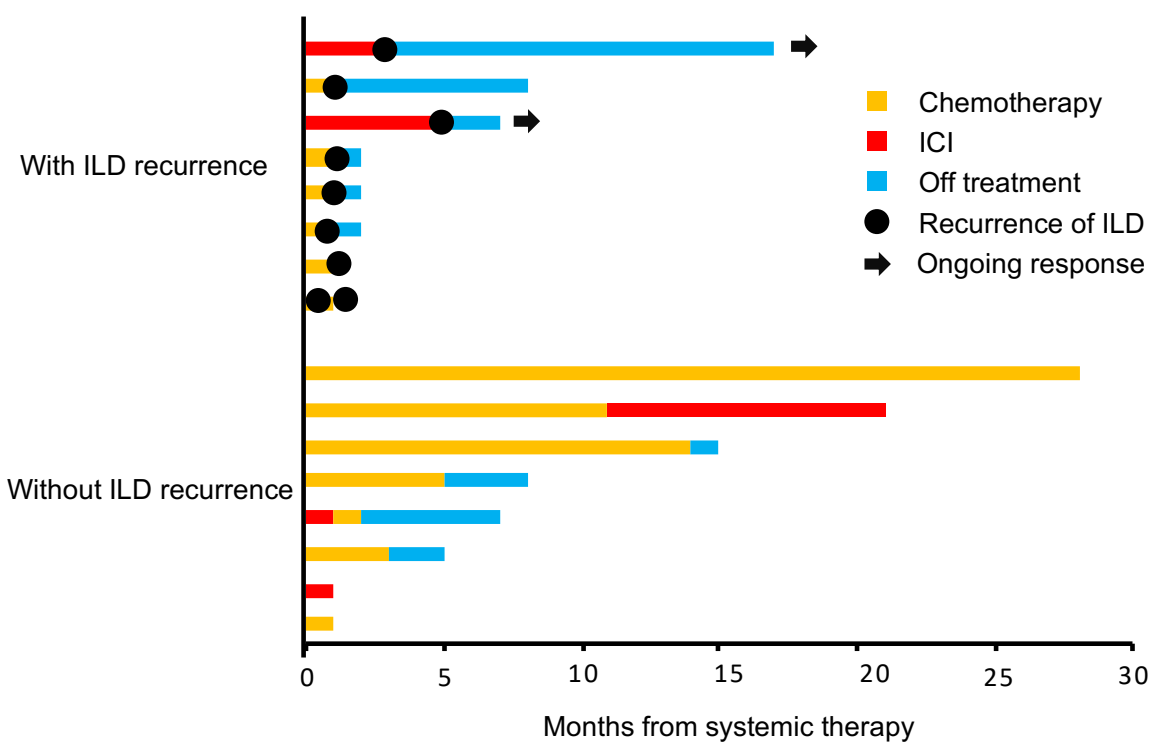

Figure 5 Duration of treatment with or without ILD recurrence after systemic cancer therapy. ILD, interstitial lung disease; ICI, immune checkpoint inhibitor.

patient recovered from ICI-related ILD and no patients experienced the recurrence of ILD in the no subsequent systemic cancer therapy group. Therefore, we considered that systemic cancer therapy following ICI-related ILD caused the recurrence of ILD. Patients did not receive subsequent systemic cancer therapy for a variety of reasons. Thirteen patients did not receive subsequent systemic cancer therapy at a physician's discretion, one patient for deterioration of PS, one patient did not recover from ILD and one patient had complete remission of NSCLC. These reasons for not treating patients with ICI-related ILD might affect their prognosis. Although a high frequency of ILD recurrence was observed, grade 2 or less ILD occurred in $78 \%$ of regimens (Table 2). Similar to the report by Simonaggio and colleagues on the severity of second irAEs, the results from our study also indicated that the severity of the second episode of ILD was not worse than that of the first (Tables 1,2). On the other hand, the current study showed that ILD recurrence was associated with a poor prognosis. Both chemotherapy and ICI rechallenge caused a second episode of ILD; however, a long response duration was observed only in patients who received ICI retreatment (Figure 5).

The current study demonstrated that patients who received subsequent systemic cancer therapies had a better prognosis than those who did not; however, patients who developed ILD recurrence had a poor prognosis
(Figures 2,3). The results from this study suggest that predictive factors for ILD recurrence are required to administer systemic cancer therapy to patients with ICI-related ILD. We previously reported that patients with a GGO had shorter survival times than those with COP (14). However, there was no difference in the risk of ILD recurrence according to radiological features, and recurrent ICI-related ILD often showed similar imaging findings (Figure 4). Three out of 16 patients in systemic cancer therapy group had interstitial lung abnormalities before initial ICI treatment (Table 1). In these 3 patients, 2 had the recurrence of ICI-related ILD after the subsequent cancer therapy. Interstitial lung abnormalities might be the risk of the recurrence of ICI-related ILD after subsequent cancer therapy.

To our knowledge, this is the first report to evaluate subsequent systemic cancer treatment after the onset of ICI-related ILD, and we believe that the results from this study will aid in future clinical practice. The limitations of the present study include the relatively small number of patients with ILD and its retrospective nature. Further study is warranted to establish an appropriate systemic cancer treatment for patients with ICI-related ILD.

\section{Conclusions}

According to the current study, systemic cancer treatment 
is effective in patients with ICI-related ILD; however, its safety is uncertain because of the high risk of ILD recurrence and poor survival outcome following ILD recurrence.

\section{Acknowledgments}

The authors thank the patients, their families, all study investigators and Hiroko Aita for their contributions to the study.

Funding: None.

\section{Footnote}

Reporting Checklist: The authors have completed the STROBE reporting checklist. Available at https://dx.doi. org/10.21037/tlcr-21-198

Data Sharing Statement: Available at https://dx.doi. org/10.21037/tlcr-21-198

Peer Review File: Available at https://dx.doi.org/10.21037/ tlcr-21-198

Conflicts of Interest: All authors have completed the ICMJE uniform disclosure form (available at https:// dx.doi.org/10.21037/tlcr-21-198). Satoshi Watanabe has received grants and personal fees from AstraZeneca, personal fees from Chugai Pharma, personal fees from Ono Pharmaceutical, personal fees from Bristol-Myers, grants and personal fees from Boehringer Ingelheim, personal fees from Eli Lilly, personal fees from MSD, personal fees from Taiho Pharmaceutical, personal fees from Pfizer, personal fees from Novartis, personal fees from Daiichi Sankyo. Takeshi Ota has received personal fees from Boehringer Ingelheim, personal fees from MSD, personal fees from Eli Lilly, personal fees from AstraZeneca, personal fees from Chugai-pharm, personal fees from Bristol-Myers Squibb. Satoshi Shoji has received personal fees from AstraZeneca, personal fees from Chugai Pharma, personal fees from Taiho Pharmaceutical, personal fees from MSD. Koichiro Nozaki has received personal fees from AstraZeneca, personal fees from Boehringer Ingelheim, personal fees from Taiho Pharmaceutical, personal fees from MSD. Kosuke Ichikawa has received personal fees from AstraZeneca, personal fees from Chugai Pharma, personal fees from Bristol-Myers, personal fees from Boehringer Ingelheim, personal fees from Ono
Pharmaceutical, personal fees from Taiho Pharmaceutical, personal fees from Novartis International AG, personal fees from Daiichi Sankyo Company. Satoshi Hokari has received personal fees from GlaxoSmithKline Inc. Masachika Hayashi has received personal fees from BoehringerIngelheim Japan, personal fees from AstraZeneca, personal fees from Taiho Pharmaceutical, personal fees from Daiichi Sankyo. Hiroyuki Ishikawa has received personal fees from AstraZeneca, personal fees from Bayer, personal fees from Daiichi Sankyo, grants from Eisai, personal fees from Fujifilm Toyama Chemical, grants from Guerbet Japan, grants and personal fees from Nihon Medi-Physics. Tetsuya Abe has received personal fees from Eli Lilly Japan, personal fees from Chugai Pharmaceutical, personal fees from Taiho Pharmaceutical, personal fees from Ono Pharmaceutical, personal fees from Bristol-Myers Squibb, personal fees from AstraZeneca, personal fees from Mylan. Satoru Miura has received personal fees from Chugai Pharmaceutical, personal fees from ONO Pharmaceutical, personal fees from AstraZeneca, personal fees from Eli Lilly, personal fees from MSD, personal fees from Boehringer Ingelheim, personal fees from Taiho Pharmaceutical, personal fees from Novartis, personal fees from Bristol-Myers Squibb, from Kyowa Hakko Kirin. Hiroshi Tanaka has received grants and personal fees from Bristol-Myers Squibb, grants and personal fees from Eli Lilly, grants and personal fees from MSD, grants and personal fees from Taiho pharmaceutical, grants and personal fees from Pfizer, grants and personal fees from Novartis, grants and personal fees from Chugai pharmaceutical, grants and personal fees from Astra Zeneca, grants and personal fees from Boehringer Ingelheim, grants and personal fees from Ono pharmaceutical, grants and personal fees from Merck. Masaaki Okajima has received personal fees from AstraZeneca, personal fees from Ono Pharmaceutical, personal fees from Bristol-Myers, personal fees from Boehringer Ingelheim, personal fees from MSD, personal fees from Taiho Pharmaceutical. Nobumasa Aoki has received personal fees from Meiji Seika Pharma, grants and personal fees from MSD. Yasuyoshi Ohshima has received personal fees from Boehringer-Ingelheim Japan, personal fees from Meiji seika pharma. Toshiyuki Koya has received personal fees from AstraZeneca, BoehringerIngelheim, Sanofi Genzyme, Novartis, Daiichi Sankyo, Kyorin Pharmaceutical and GlaxoSmithKline. Toshiaki Kikuchi has received grants and personal fees from Chugai Pharma, grants and personal fees from Boehringer Ingelheim, grants and personal fees from Eli Lilly, grants and personal fees from MSD K.K., personal fees from 
Astellas Pharma Inc., grants and personal fees from Taiho Pharmaceutical CO., Ltd., personal fees from BristolMyers Squibb Company, personal fees from Pfizer Japan Inc., grants and personal fees from Daiichi Sankyo CO., Ltd., personal fees from Taisho Toyama Pharmaceutical Co., Ltd., personal fees from Janssen Pharmaceutical K.K., personal fees from Japan BCG Laboratory, grants and personal fees from Ono Pharmaceutical Co., Ltd., personal fees from Novartis Pharma K.K., personal fees from Mylan N.V., grants and personal fees from AstraZeneca, personal fees from Roche Diagnostics K.K., grants and personal fees from Shionogi \& Co., Ltd., grants from TEIJIN PHARMA Ltd., grants from KYORIN Pharmaceutical Co., Ltd. The other authors have no conflicts of interest to declare.

Ethical Statement: The authors are accountable for all aspects of the work in ensuring that questions related to the accuracy or integrity of any part of the work are appropriately investigated and resolved. The study was conducted in accordance with the Declaration of Helsinki (as revised in 2013). The study was approved by institutional review board of Niigata University (the registration number: 2017-0295) and each participating institution. Individual consent for this retrospective analysis was waived.

Open Access Statement: This is an Open Access article distributed in accordance with the Creative Commons Attribution-NonCommercial-NoDerivs 4.0 International License (CC BY-NC-ND 4.0), which permits the noncommercial replication and distribution of the article with the strict proviso that no changes or edits are made and the original work is properly cited (including links to both the formal publication through the relevant DOI and the license). See: https://creativecommons.org/licenses/by-nc-nd/4.0/.

\section{References}

1. Herbst RS, Baas P, Kim DW, et al. Pembrolizumab vs. docetaxel for previously treated, PD-L1-positive, advanced non-small-cell lung cancer (KEYNOTE-010): a randomised controlled trial. Lancet 2016;387:1540-50.

2. Brahmer J, Reckamp KL, Baas P, et al. Nivolumab vs. Docetaxel in Advanced Squamous-Cell Non-Small-Cell Lung Cancer. N Engl J Med 2015;373:123-35.

3. Borghaei H, Paz-Ares L, Horn L, et al. Nivolumab vs. Docetaxel in Advanced Nonsquamous Non-Small-Cell Lung Cancer. N Engl J Med 2015;373:1627-39.

4. Horn L, Mansfield AS, Szczęsna A, et al. First-Line
Atezolizumab plus Chemotherapy in Extensive-Stage Small-Cell Lung Cancer. N Engl J Med 2018;379:2220-9.

5. Paz-Ares L, Dvorkin M, Chen Y, et al. Durvalumab plus platinum-etoposide vs. platinum-etoposide in firstline treatment of extensive-stage small-cell lung cancer (CASPIAN): a randomised, controlled, open-label, phase 3 trial. Lancet 2019;394:1929-39.

6. Postow MA, Sidlow R, Hellmann MD. Immune-Related Adverse Events Associated with Immune Checkpoint Blockade. N Engl J Med 2018;378:158-68.

7. Naidoo J, Page DB, Li BT, et al. Toxicities of the antiPD-1 and anti-PD-L1 immune checkpoint antibodies. Ann Oncol 2015;26:2375-91.

8. Ksienski D, Wai ES, Croteau N, et al. Efficacy of Nivolumab and Pembrolizumab in Patients With Advanced Non-Small-Cell Lung Cancer Needing Treatment Interruption Because of Adverse Events: A Retrospective Multicenter Analysis. Clin Lung Cancer 2019;20:e97-e106.

9. Haratani K, Hayashi H, Chiba Y, et al. Association of Immune-Related Adverse Events With Nivolumab Efficacy in Non-Small-Cell Lung Cancer. JAMA Oncol 2018;4:374-8.

10. Shafqat H, Gourdin T, Sion A. Immune-related adverse events are linked with improved progression-free survival in patients receiving anti-PD-1/PD-L1 therapy. Semin Oncol 2018;45:156-63.

11. Tamura N, Horinouchi H, Sekine K, et al. Efficacy of subsequent docetaxel +/- ramucirumab and S-1 after nivolumab for patients with advanced non-small cell lung cancer. Thorac Cancer 2019;10:1141-8.

12. Yoshimura A, Yamada T, Okuma Y, et al. Retrospective analysis of docetaxel in combination with ramucirumab for previously treated non-small cell lung cancer patients. Transl Lung Cancer Res 2019;8:450-60.

13. Simonaggio A, Michot JM, Voisin AL, et al. Evaluation of Readministration of Immune Checkpoint Inhibitors After Immune-Related Adverse Events in Patients With Cancer. JAMA Oncol 2019;5:1310-7.

14. Watanabe S, Ota T, Hayashi M, et al. Prognostic significance of the radiologic features of pneumonitis induced by anti-PD-1 therapy. Cancer Med 2020;9:3070-7.

15. Maemondo M, Inoue A, Kobayashi K, et al. Gefitinib or chemotherapy for non-small-cell lung cancer with mutated EGFR. N Engl J Med 2010;362:2380-8.

16. Pellegrino B, Facchinetti F, Bordi P, et al. Lung Toxicity in Non-Small-Cell Lung Cancer Patients Exposed 
to ALK Inhibitors: Report of a Peculiar Case and Systematic Review of the Literature. Clin Lung Cancer 2018;19:e151-61.

17. Su Q, Zhu EC, Wu JB, et al. Risk of Pneumonitis and Pneumonia Associated With Immune Checkpoint Inhibitors for Solid Tumors: A Systematic Review and Meta-Analysis. Front Immunol 2019;10:108.

18. Fujimoto D, Kato R, Morimoto T, et al. Characteristics and Prognostic Impact of Pneumonitis during Systemic Anti-Cancer Therapy in Patients with Advanced NonSmall-Cell Lung Cancer. PLoS One 2016;11:e0168465.

19. Nakagawa K, Kudoh S, Ohe Y, et al. Postmarketing surveillance study of erlotinib in Japanese patients with non-small-cell lung cancer (NSCLC): an interim analysis of 3488 patients (POLARSTAR). J Thorac Oncol 2012;7:1296-303.

Cite this article as: Sato Y, Watanabe S, Ota T, Kushiro K, Fujisaki T, Takahashi M, Ohtsubo A, Shoji S, Nozaki K, Ichikawa K, Hokari S, Kondo R, Hayashi M, Ishikawa H, Miyabayashi T, Abe T, Miura S, Tanaka H, Okajima M, Terada M, Ishida T, Iwashima A, Sato K, Yoshizawa H, Aoki N, Ohshima Y, Koya T, Kikuchi T. Subsequent systemic therapy for non-small cell lung cancer patients with immune checkpoint inhibitor-related interstitial lung disease. Transl Lung Cancer Res 2021;10(7):3132-3143. doi: 10.21037/tlcr-21-198
20. Sata M, Sasaki S, Oikado K, et al. Treatment and relapse of interstitial lung disease in nivolumab-treated patients with non-small cell lung cancer. Cancer Sci 2021;112:1506-13.

21. Huang Y, Fan H, Li N, et al. Risk of immune-related pneumonitis for PD1/PD-L1 inhibitors: Systematic review and network meta-analysis. Cancer Med 2019;8:2664-74.

22. Kashiwabara K, Semba H, Fujii S, et al. Re-administration of Chemotherapy in Patients with Advanced Non-small Cell Lung Cancer Who Recovered from Chemotherapyinduced Interstitial Lung Disease. In Vivo 2018;32:851-7.

23. Kashiwabara K, Semba H, Fujii S, et al. Outcome in advanced non-small cell lung cancer patients with successful rechallenge after recovery from epidermal growth factor receptor tyrosine kinase inhibitor-induced interstitial lung disease. Cancer Chemother Pharmacol 2017;79:705-10. 


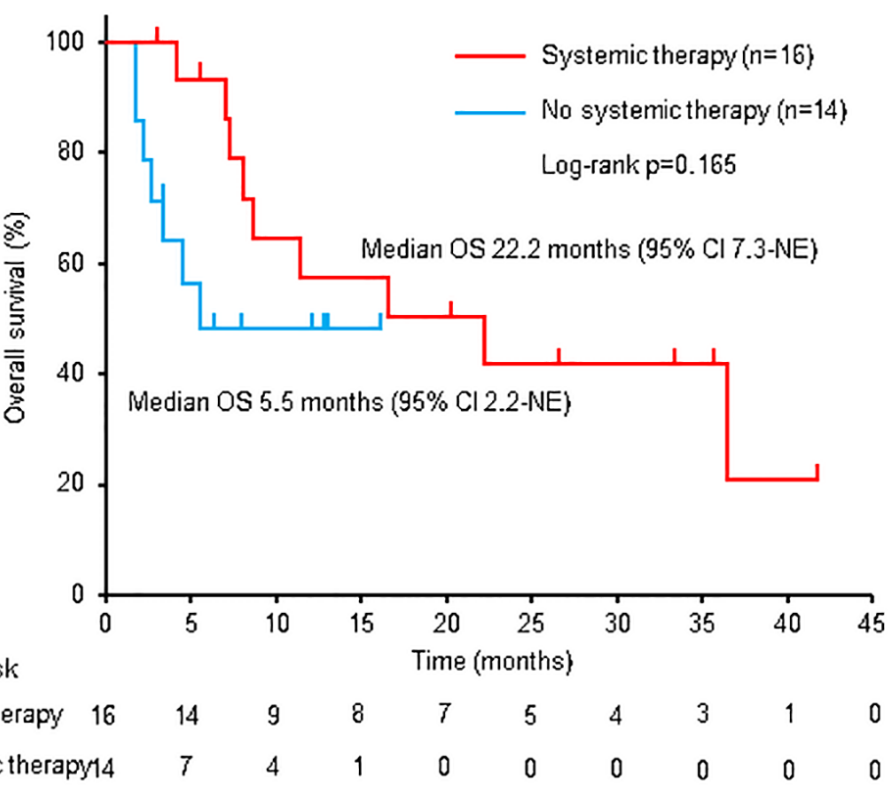

Figure S1 Six-week landmark analysis for overall survival in patients with or without systemic cancer therapy after the 1st episode of ICIrelated ILD. ILD, interstitial lung disease; OS, overall survival; CI, confidence interval; NE, not evaluable. 
Table S1 Baseline characteristics of patients with and without the recurrent ICI-related ILD

\begin{tabular}{|c|c|c|c|c|}
\hline \multirow{2}{*}{\multicolumn{2}{|c|}{ Characteristic }} & Recurrent ICI-related ILD & No recurrent ILD & \multirow{2}{*}{$P$ value } \\
\hline & & $(\mathrm{N}=8)$ & $(\mathrm{N}=8)$ & \\
\hline Median age (range), years & & $65(62-73)$ & $65(45-74)$ & $0.33^{\mathrm{a}}$ \\
\hline \multirow[t]{2}{*}{ Sex, n (\%) } & Male & $7(88)$ & $5(63)$ & $0.25^{\mathrm{b}}$ \\
\hline & Female & $1(12)$ & $3(37)$ & \\
\hline \multirow[t]{2}{*}{ Smoking status, n (\%) } & Current or former & $7(88)$ & $6(75)$ & $0.52^{\mathrm{b}}$ \\
\hline & Never & $1(12)$ & $2(25)$ & \\
\hline \multirow[t]{4}{*}{ ECOG-PS, n (\%) } & 0 & $1(12)$ & $3(37)$ & $0.39^{b}$ \\
\hline & 1 & $5(63)$ & $5(63)$ & \\
\hline & $\geq 2$ & $1(12)$ & 0 & \\
\hline & Unknown & $1(12)$ & 0 & \\
\hline \multirow[t]{3}{*}{ Disease stage, n (\%) } & IIIB & 0 & 0 & $0.05^{\mathrm{b}}$ \\
\hline & IV & $2(25)$ & $6(75)$ & \\
\hline & Relapse after local therapy & $6(75)$ & $2(25)$ & \\
\hline \multirow[t]{3}{*}{ Histology, n (\%) } & Adenocarcinoma & $2(25)$ & $2(25)$ & $1^{\mathrm{b}}$ \\
\hline & Squamous carcinoma & $5(63)$ & $5(63)$ & \\
\hline & Others & $1(12)$ & $1(12)$ & \\
\hline \multirow[t]{3}{*}{ Line of anti-PD-1 therapy, $n$ (\%) } & 1 & $2(25)$ & 0 & $0.31^{b}$ \\
\hline & 2 & $4(50)$ & $5(63)$ & \\
\hline & 3 & $2(25)$ & $3(37)$ & \\
\hline \multirow[t]{4}{*}{ PD-L1 expression, n (\%) } & $<1$ & $1(12)$ & 0 & $0.16^{\mathrm{b}}$ \\
\hline & $1-49 \%$ & 0 & 0 & \\
\hline & $>50 \%$ & $2(25)$ & 0 & \\
\hline & Unknown & $5(63)$ & $8(100)$ & \\
\hline \multirow[t]{2}{*}{ Anti-PD-1 therapy, $\mathrm{n}(\%)$} & Nivolumab & $6(75)$ & $8(100)$ & $0.13^{\mathrm{b}}$ \\
\hline & Pembrolizumab & $2(25)$ & 0 & \\
\hline \multirow[t]{5}{*}{ Response to anti-PD-1 therapy } & $\mathrm{CR}$ & $1(12)$ & $1(12)$ & $0.86^{\mathrm{b}}$ \\
\hline & PR & $2(25)$ & $2(25)$ & \\
\hline & SD & $3(37)$ & $3(37)$ & \\
\hline & PD & $1(12)$ & $2(25)$ & \\
\hline & NE & $1(12)$ & 0 & \\
\hline \multicolumn{2}{|c|}{ Baseline interstitial lung abnormality, $\mathrm{n}(\%)$} & $2(25)$ & $1(12)$ & $0.55^{\mathrm{a}}$ \\
\hline \multirow[t]{2}{*}{ Grade of initial ILD } & $1-2$ & $4(50)$ & $7(88)$ & $0.11^{b}$ \\
\hline & $3-4$ & $4(50)$ & $1(12)$ & \\
\hline \multirow[t]{3}{*}{ Radiologic features, n (\%) } & COP-like & $4(50)$ & $5(63)$ & $0.61^{\mathrm{b}}$ \\
\hline & GGO & $4(50)$ & $3(37)$ & \\
\hline & Not otherwise specified & 0 & 0 & \\
\hline Systemic steroid use, n (\%) & & $7(88)$ & $6(75)$ & $0.55^{\mathrm{a}}$ \\
\hline Time to the ILD, days (range) & & $24(5-523)$ & $50.5(1-295)$ & $0.56^{\mathrm{a}}$ \\
\hline
\end{tabular}

Differences between groups were identified using ${ }^{a}$ student's $t$ test or ${ }^{b} \mathrm{Chi}-S q u a r e$ test. ICI, immune checkpoint inhibitor; ILD, interstitial lung disease; ECOG, Eastern Cooperative Oncology Group; PS, performance status; PD-1, programmed cell death-1; PD-L1, PD-ligand 1; CR, complete response; PR, partial response; SD, stable disease; PD, progressive disease; NE, not evaluable; COP, cryptogenic organizing pneumonia; GGO, ground glass opacity. 